\title{
Global neurosurgery: continued momentum at the 72nd World Health Assembly
}

\author{
Gail Rosseau, MD, ${ }^{1}$ Walter D. Johnson, MD, MBA, MPH, ${ }^{2}$ Kee B. Park, MD, MPH, ${ }^{3}$ \\ Peter J. Hutchinson, MBBS, FMedSci, ${ }^{4}$ Laura Lippa, MD, ${ }^{5}$ Russell Andrews, MD, ${ }^{6}$ \\ Franco Servadei, MD, ${ }^{7}$ and Roxanna M. Garcia, MD, MS, MPH ${ }^{8}$
}

\begin{abstract}
1Department of Neurosurgery, George Washington University School of Medicine and Health Sciences, Washington, DC; ${ }^{2}$ Emergency and Essential Surgical Care Programme, World Health Organization, Geneva, Switzerland; ${ }^{3}$ Global Neurosurgery Initiative, Program in Global Surgery and Social Change, Harvard Medical School, Boston, Massachusetts; ${ }^{4}$ Department of Neurosurgery, University of Cambridge, United Kingdom; ${ }^{5}$ Department of Neurosurgery, Azienda Ospedaliera Universitaria Senese, Siena, Italy; ${ }^{6}$ Nanotechnology and Smart Systems, NASAAmes Research Center, Mountain View, California; ${ }^{7}$ Department of Neurosurgery, Humanitas University, Milan, Italy; and ${ }^{8}$ Department of Neurosurgery, McGaw School of Medicine, Northwestern University, Chicago, Illinois
\end{abstract}

Global neurosurgery is the practice of neurosurgery with the primary purpose of delivering timely, safe, and affordable neurosurgical care to all who need it. This field is led by neurosurgeons, and global neurosurgery sessions are now part of every major international neurosurgical meeting. The World Federation of Neurosurgical Societies (WFNS) is working to coordinate activities and align all related activities for greater impact. This report updates the contributions made by the WFNS-WHO Liaison Committee at the most recent World Health Assembly (WHA) in 2019. The WHA is a decisionmaking body of the World Health Organization (WHO), attended by its 194 Member States. The WFNS has maintained official relations as a nongovernmental organization with the WHO for over 30 years, and this year 15 neurosurgical delegates attended events during the WHA. Participation by neurosurgeons continues to grow as many WHA events focused on global surgery have intrinsically involved neurosurgical leadership and participation. This year, resolution WHA72.31, entitled "Emergency and trauma care, Emergency care systems for universal health coverage: ensuring timely care for the acutely ill and injured," was passed. This resolution provides further opportunities for neurosurgical advocacy as the landscape of global surgery gains recognition and momentum.

https://thejns.org/doi/abs/10.3171/2019.11.JNS191823

KEYWORDS global neurosurgery; global surgery; workforce; capacity; World Health Assembly; World Health Organization

$\mathrm{T}$ HE World Health Assembly (WHA), a forum for the decision-making body of the World Health Organization (WHO), is attended by its 194 Member States and focuses on specific agenda items prepared by the Executive Board. It occurs annually to determine policies within the WHO, appoint the director-general, supervise finances for global health priorities, and approve program budgets. Last year, we reported a summary of the WHA and the significant momentum that continues to build around the field of global neurosurgery. ${ }^{14}$ Global surgery as a formal area of universal healthcare coverage (UHC) has continued to gain attention since 2015, when The Lancet Commission on Global Surgery Report was released, shortly followed by the unanimous adoption of the WHA resolution WHA68.15, "Strengthening emergency and essential surgical care and anaesthesia as a component of universal health coverage." 10,15 Since that time, global surgery as a public health priority has been gaining recognition and attention from key global leaders and stakeholders in public health. Again this year, the World Federation of Neurosurgical Societies (WFNS; www.wfns.org) coordinated a neurosurgical delegation composed of 15 volunteer international neurosurgeons to advocate for universal access to lifesaving or disability-averting neurosurgical care. The following report highlights the participation of this neurosurgical delegation at the 72nd WHA in advocacy of

ABBREVIATIONS AANS = American Association of Neurological Surgeons; EANS = European Association of Neurosurgical Societies; EESC = Emergency and Essential Surgical Care; FIENS = Foundation for International Education in Neurological Surgery; HIC = high-income country; LMICs = low- and middle-income countries; NSOAP = national surgical, obstetric, and anesthesia plan; SDG = Sustainable Development Goal; UHC = universal healthcare coverage; WFNS = World Federation of Neurosurgical Societies; WHA = World Health Assembly; WHO = World Health Organization .

SUBMITTED July 1, 2019. ACCEPTED November 15, 2019.

INCLUDE WHEN CITING Published online January 17, 2020; DOI: 10.3171/2019.11.JNS191823. 
global neurosurgery. Political leaders in all United Nations Member States consider strengthening surgical systems in the developing world to be essential in order to reach the 17 agreed-upon United Nations Sustainable Development Goals (SDGs) and their targets by 2030. Fourteen among the 17 SDGs have specific relevance to neurosurgical care and providers, thus calling for ample neurosurgical advocacy and participation to create improved health equity. ${ }^{1}$

\section{2nd World Health Assembly Delegation}

The WFNS has maintained official relations as a nongovernmental organization with the WHO for over 30 years, yet regular attendance and participation by its Member States have been limited. The delegation attending this year included neurosurgeons from Switzerland, the United Kingdom, the Philippines, Italy, and the United States, as well as the current WFNS president. Importantly, three neurosurgery trainees were able to attend the WHA as part of the WFNS delegation over the last 2 years, which represents an important influence in early career development for young neurosurgeons pursuing global neurosurgery. Additionally, several members over the last 3 years have been able to attend annually, which contributes to building important partnerships with other advocacy groups interested in advancing the agenda for surgery, obstetrics, and anesthesia as a component of UHC.

\section{Global Neurosurgery Advocacy at the WHA}

Prior to the WHA, the G4 Alliance (www.theg4 alliance.org) meets each year in Geneva to prepare its four so-called SOTA (surgery/obstetrics/trauma/anesthesia) member groups for collaborative advocacy. The G4 Alliance Advocacy and Policy Working Group identifies key areas for policy development and partnership in global surgery. This group is led by a senior neurosurgeon and, as its first assignment, is preparing for an enhanced international focus on folate fortification to prevent folic acidpreventable spina bifida and anencephaly (FAP SBA).

The WHA opened on May 20, 2019, beginning with a framework for priority areas in modern global health practice. The editor-in-chief of The Lancet, Dr. Richard Horton, addressed the assembly and identified access to safe and affordable surgical care as one of his top five recommendations. The WHO director-general, Dr. Tedros Adhanom Ghebreyesus, formally recognized the need for surgical, obstetric, and anesthesia care as necessary for achieving UHC and reaching the targets set in the 17 SDGs, especially those targets in SDG3 that will only be achieved with improved access to surgical care.

Neurosurgeons participated in WHA side events that included the following topic areas: addressing global surgery in the context of UHC, the global workforce deficit, priorities in epilepsy, emergency care systems for UHC, strengthening partnerships for health systems, access to essential medicines, surgical patient safety and quality, surgery in the context of SDGs, and safe and affordable surgery. There were 12 events with relevance to surgery, obstetric, and anesthesia care, one of which was an official WHA side event hosted by several health ministers who are surgeons. A day-long technical meeting was hosted by neurosurgeon Walter Johnson, lead of the WHO Emergency and Essential Surgical Care (EESC) Programme. The speakers included four neurosurgeons who presented on important collaborations; the development of partnerships in surgical systems; scaling up surgical, obstetric, and anesthesia services; and regional updates for programs actively defining surgical capacity in-country. Establishing working relationships with colleagues in global surgery was an essential component of these daily events, including professional associations, Ministries of Health, and nonprofit organizations. As low- and middle-income countries (LMICs) develop national surgical, obstetric, and anesthesia plans (NSOAPs), local neurosurgical participation in providing expert input into the planning process at the country level is essential. It is crucial that neurosurgeons have both a presence and a leadership role in identifying and championing the path to improve surgical capacity throughout the world, as it was estimated that the need for 5 million emergency and essential neurosurgical operations is currently unmet, mostly in the developing world. ${ }^{10}$ Additionally, formalizing a structured and transparent process of neurosurgical advocacy, metrics to measure the impact of such activities, and the landscape of global surgery and neurotrauma service delivery should be a priority as the field advances. It is the role of the WFNS to bring research, implementation, policy engagement, and advocacy efforts together and align them under a common vision of universal access to lifesaving or disability-averting neurosurgical care. A WFNS Global Neurosurgery Action Plan will help neurosurgeons become more strategic in their efforts and enhance the specialty's impact as it continues with the WHO-related activities, such as the WHA.

\section{WHA Resolutions and Opportunities for Advocacy}

On May 27, 2019, resolution WHA72.31, entitled "Emergency and trauma care, Emergency care systems for universal health coverage: ensuring timely care for the acutely ill and injured," was passed..$^{16}$ This resolution emphasizes the need for urgent access and calls for the strengthening of emergency care systems as an essential component of UHC. The emergency care systems include the range of services delivered at the scene of the event (prehospital system) to arrival at facilities with operative and critical care capabilities, with time as a dimension of quality. This appropriately follows several other important WHA resolutions targeting advancements in surgical care (Table 1) and further provides a mandate to allow LMICs and resource-poor settings to prioritize assessment, development, and restructuring of healthcare systems aimed at caring for acutely injured patients at the time of the event and for those whose illness requires urgent care. For example, neurosurgeons continue to be fundamental in international advocacy of patients suffering from traumatic brain injury (TBI) and spinal cord injury (SCI), which represent significant contributors to global morbidity and mortality. TBI and SCI correspond to approximately 55 million (estimated range 53-58 million) and 27 million (estimated range 25-30 million) prevalent cases, respec- 
TABLE 1. WHA resolution descriptions focused on emergency and trauma care

\begin{tabular}{|c|c|}
\hline $\begin{array}{c}\text { WHA } \\
\text { Resolution (yr) }\end{array}$ & Description \\
\hline $56.24(2003)$ & $\begin{array}{l}\text { Implementing the recommendation of the "World } \\
\text { report on violence and health" }\end{array}$ \\
\hline $57.10(2004)$ & Road safety \& health \\
\hline $60.22(2007)$ & Health systems: emergency care systems \\
\hline $64.10(2011)$ & $\begin{array}{l}\text { Strengthening national health emergency \& disaster } \\
\text { management capacities \& resilience of health } \\
\text { systems }\end{array}$ \\
\hline $68.15(2015)$ & $\begin{array}{l}\text { Strengthening emergency \& essential surgical care } \\
\& \text { anesthesia as a component of universal health } \\
\text { coverage }\end{array}$ \\
\hline $69.1(2016)$ & $\begin{array}{l}\text { Strengthening essential public health functions in } \\
\text { support of the achievement of universal health } \\
\text { coverage }\end{array}$ \\
\hline $78 / 271(2018)$ & Improving global road safety \\
\hline $72.31(2019)$ & $\begin{array}{l}\text { Emergency \& trauma care, emergency care systems } \\
\text { for universal health coverage: ensuring timely care } \\
\text { for the acutely ill \& injured }\end{array}$ \\
\hline
\end{tabular}

Resolutions passed since 2003 to the present, addressing emergency care and surgical services as a component of UHC.

tively, ${ }^{8}$ and remain a priority in achieving UHC and context-specific priority areas in global neurosurgery, which relies heavily on advocacy by neurosurgeons.

Currently, the neurosurgical community lacks a unified, formalized framework for participation in neurosurgical global health advocacy, policy, and research. Some of the barriers that prevent full engagement from neurosurgeons, mostly in high-income countries (HICs), have been described ${ }^{6}$ and require systematic restructuring to permit a subset of our neurosurgical colleagues to become leaders in making neurosurgical care available to the most vulnerable populations internationally. This begins early in the educational process but must be championed and supported internally through residency training, in early faculty development, and as seasoned neurosurgical academicians and practitioners. These changes must be valued and encouraged by key neurosurgery leadership in order for leaders to emerge from within our community. Below we provide several emerging examples of advocacy but also highlight specific priority areas (Fig. 1) that would allow for more focused advocacy and involvement as the movement of global surgery evolves.

The American Association of Neurological Surgeons (AANS; www.aans.org) has sponsored global neurosurgery sessions at its annual meeting the past 3 years and has offered a course in global neurosurgical practice for those who wish to hone their skills for operating and teaching in LMICs. The WFNS and European Association of Neurosurgical Societies (EANS; www.eans.org) are cosponsoring a similar course in Geneva in 2020. The course Neurosurgery Around the World: Education and Other Opportunities has been offered by the WFNS in association with AANS. The Harvard Program in Global Surgery and Social Change (https://www.pgssc.org) has mentored and sponsored medical students, as well as neurosurgery trainees and faculty, to be involved in contemporary global health research and advocacy, several of whom have spent time working with the WHO EESC Programme. Additionally, the National Institutes of Health (NIH) Fogarty International Center and the Gates Foundation are sponsoring research for young trainees interested in careers in global neurosurgery. The University of Toronto has outlined a tenure track in global surgery and global neurosurgery (https://surgery.utoronto.ca/global-surgery). Funding remains inadequate for global surgery research initiatives as a whole, despite the fact that $11 \%-30 \%$ of the global burden of disease is surgical. It has been reported that only $4.1 \%$ of global health research activity is surgical, and of all surgical research worldwide, only $4.3 \%$ is relevant to underserved populations. ${ }^{12}$ Major efforts are in place to remedy these differences, but initiatives to garner more funding for rigorous scientific research in global surgery must be prioritized and rigorously pursued.

Fostering and promoting translational research and surgical skills training that focuses on sustainable capacity building between HICs and LMICs are in all stages of development. The documented significant disparities in the neurosurgical workforce ${ }^{4,9,11,13}$ create challenges to building capacity for neurosurgical education and care. Rigorous data collection and research skills may be limited in some LMICs, but capacity and telemedicine in HICs can partially bridge this gap. The bilateral exchange of such information may be of benefit in HICs, where widespread inequities in access to care are known to exist. ${ }^{5}$ Neurosurgeons all over the world are linked by a common commitment to provide care for some of the most vulnerable patients in the healthcare system. Collaboratively working to further existing relationships and creating avenues for new sustainable bidirectional relationships in the delivery of essential neurosurgical care and research are critical components of advocacy for the health and well-being of our patients and the protection of vulnerable populations. ${ }^{2,3}$

\section{Global Neurosurgery, the World Health Assembly, and Beyond}

Today, as the global surgery movement expands, the importance of the WFNS's role in advocating for universal access to neurosurgical care and providing technical and strategic input for the WHO and the Ministries of Health cannot be understated. Thus, the significance of the WHA, where all Ministers of Health convene, is unparalleled. Yet, the neurosurgical community's interfacing with global health governance, policymakers, donors, and other stakeholders lacks a unified, formalized framework for participation in neurosurgical global health advocacy, policy, and research. There is an urgent need for the neurosurgical community, represented by the WFNS, to respond with a clear strategy, a unifying goal, a set of guiding principles, and an execution plan-A Global Neurosurgery Action Plan.

It is well known that neurosurgeons have been involved in improving neurosurgical care in the developing world for decades. For example, since 2000, the WFNS Founda- 
- Involvement in national/regional/international organized global health, non-communicable disease groups, and global surgery

- Mobilize neurosurgical support and leadership

- Neurosurgeons assume leadership positions in global health organizations

- Ethical coordination with industry and faith-based partners

\footnotetext{
- Create disease- and process-specific metrics in global neurosurgery outcomes

- Foster collaboration \& transparency in international relationships

- Use evaluation results to improve context-specific neurosurgical issues through continuous implementation and planning teams

- Implement specific, measurable, achievable, relevant and timely (SMART) goals for evaluation
}

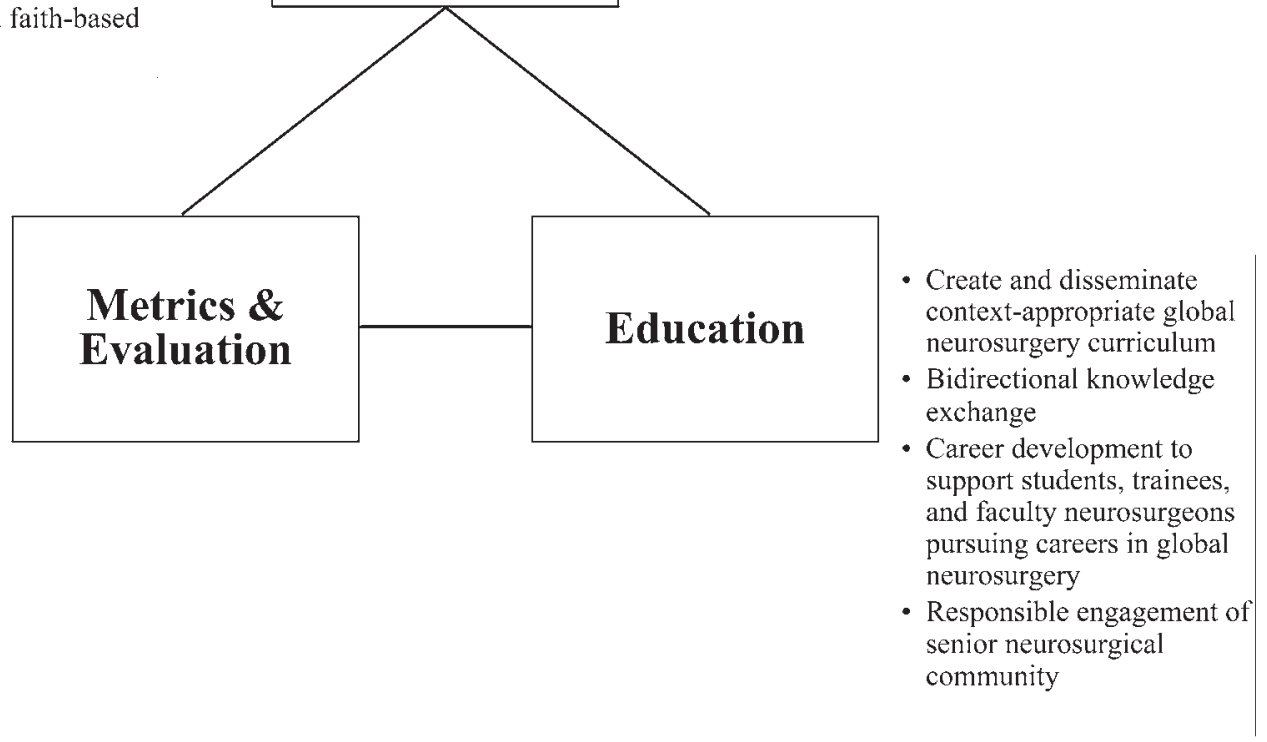

FIG. 1. Priorities in global neurosurgery should encompass outreach and collaboration, education, and a process for metrics and evaluation to advance safe, timely, and affordable care in global surgery. Priorities should be guided by specific, measurable, achievable, relevant, and timely (SMART) evaluation criteria for each context of implementation.

tion has made a significant impact on the increase in the number of neurosurgeons and the provision of neurosurgical equipment in the developing world. The Foundation for International Education in Neurological Surgery (FIENS; $\mathrm{http} / / / \mathrm{www}$.fiens.org) has been supporting training for several decades. There are countless other organizations and individuals involved in training efforts. A key question today is whether these training activities are integrated into the NSOAPs or national surgical plans. As of this writing, 44 countries in the developing world are in various stages of developing strategic plans for strengthening surgical care, including the surgical workforce. A coordinated and sustainable effort for improving access to neurosurgical education and care will require the neurosurgical community to address each nation's local planning priorities (e.g., how many neurosurgeons will be trained, what qualifications/competencies are expected, delineating the complements to neurosurgical training activities to context-specific settings, etc.).

Major neurosurgical organizations such as the WFNS, AANS, and EANS recognize the importance of addressing neurosurgical equity by including and highlighting global neurosurgery during their annual meetings. The programs of individuals or institutions do not reflect a coordinated effort by the profession as a whole. Training is one important aspect of global neurosurgery, as is the identification of research gaps in the delivery of neurosurgical care, areas of policy engagement, implementation strategies, and advocacy.

New global neurosurgery initiatives are appearing ev- ery year. The AANS course on global neurosurgical practice that is held at its annual meetings for those who wish to hone their skills for operating and teaching in LMICs continues to be supported. Other organizations are developing similar courses. InterSurgeon (https://intersurgeon. org) is an interactive website designed to bring individuals and organizations together in partnerships to improve surgical care globally and has the potential to dramatically increase training activity. FIENS created a travel fellowship, called the Bassett Global Neurosurgery Fellowship, to support young neurosurgeons from LMICs, and the Harvard Program in Global Surgery and Social Change continues to provide intensive international opportunities for global neurosurgery research. These initiatives will benefit from a clear Global Neurosurgery Action Plan, which is in the process of creation. The goal of this action plan will be to create coherent strategies toward the intended outcome-the delivery of timely, safe, and affordable neurosurgical care to all who need it.

\section{Conclusions}

The 72nd WHA was held in Geneva, Switzerland, on May 20-28, 2019. This year, the WFNS-WHO Liaison Committee organized a delegation of 15 volunteer neurosurgeons, who led and participated in a spectrum of events related to advocacy for the neglected neurosurgical patient and for the importance of neurosurgery within the global surgery movement. ${ }^{7}$ Continued advocacy for greater global attention to neurosurgical education, training and re- 
search, as well as for the prevention and treatment of neurosurgical diseases, is necessary to help foster sustainable improvements in access to, and quality of, care for neurosurgical patients. A Global Neurosurgery Action Plan is needed to coordinate and align all related activities to maximize impact and ultimately ensure that neurosurgical care is available to all populations throughout the world.

\section{Acknowledgments}

We are grateful to the additional members of the neurosurgical team who, like us, traveled at their own expense to participate in WHA 2019: Ernest Barthelemy, Andrea Bartoli, Dylan Griswold, William Harkness, Marc Levivier, Richard Moser, Lynne Lourdes N. Lucena, and Karl Schaller. In addition, Ms. Teresa Chen and Ms. Ling Yi from the WFNS Central Office in Nyon, Switzerland, provided administrative support for registration with the WHO office for non-state actors.

Mr. Hutchinson is supported by the National Institute for Health Research (NIHR) Global Health Research Group on Neurotrauma (http://neurotrauma.world), NIHR Research Professorship, and NIHR Cambridge Biomedical Research Centre. Dr. Garcia is supported by the NIH NINDS StrokeNet grant no. U24-NS107233-01 and Fogarty Global Health Research Fellowships grant no. D43 TW010543.

\section{References}

1. Barthélemy EJ, Park KB, Johnson W: Neurosurgery and sustainable development goals. World Neurosurg 120:143-152, 2018

2. Corley J, Lepard J, Barthélemy E, Ashby JL, Park KB: Essential neurosurgical workforce needed to address neurotrauma in low- and middle-income countries. World Neurosurg 123:295-299, 2019

3. Corley J, Park KB, Johnson W: Neurosurgeons push for policy change to reduce deaths and disabilities from head and spine injuries from road traffic injuries. UN Special. ( https:// www.unspecial.org/2019/06/road-traffic-injuries/) [Accessed November 25, 2019]

4. Dewan MC, Rattani A, Fieggen G, Arraez MA, Servadei F, Boop FA, et al: Global neurosurgery: the current capacity and deficit in the provision of essential neurosurgical care. $\mathbf{J}$ Neurosurg 130:1055-1064, 2019

5. Dickman SL, Himmelstein DU, Woolhandler S: Inequality and the health-care system in the USA. Lancet 389:14311441, 2017

6. Fallah PN, Bernstein M: Barriers to participation in global surgery academic collaborations, and possible solutions: a qualitative study. J Neurosurg 130:1157-1165, 2019

7. Farmer PE, Kim JY: Surgery and global health: a view from beyond the OR. World J Surg 32:533-536, 2008

8. GBD 2016 Traumatic Brain Injury and Spinal Cord Injury Collaborators: Global, regional, and national burden of traumatic brain injury and spinal cord injury, 1990-2016: a systematic analysis for the Global Burden of Disease Study 2016. Lancet Neurol 18:56-87, 2019
9. Griswold D, Benet A, Berger MS, Lawton MT: Meeting the unmet need: training general surgeons to perform life-saving neurosurgical procedures in low-resource settings. World Neurosurg 93:474, 2016

10. Meara JG, Leather AJM, Hagander L, Alkire BC, Alonso N, Ameh EA, et al: Global Surgery 2030: evidence and solutions for achieving health, welfare, and economic development. Lancet 386:569-624, 2015

11. Mukhopadhyay S, Punchak M, Rattani A, Hung YC, Dahm J, Faruque S, et al: The global neurosurgical workforce: a mixed-methods assessment of density and growth. J Neurosurg 130:1142-1148, 2019

12. Ng-Kamstra JS, Greenberg SLM, Abdullah F, Amado V, Anderson GA, Cossa M, et al: Global Surgery 2030: a roadmap for high income country actors. BMJ Glob Health 1:e000011, 2016

13. Park KB, Johnson WD, Dempsey RJ: Global neurosurgery: the unmet need. World Neurosurg 88:32-35, 2016

14. Rosseau G, Johnson WD, Park KB, Arráez Sánchez M, Servadei F, Vaughan KA: Global neurosurgery: current and potential impact of neurosurgeons at the World Health Organization and the World Health Assembly. Executive summary of the World Federation of Neurosurgical Societies-World Health Organization Liaison Committee at the 71st World Health Assembly. Neurosurg Focus 45(4):E18, 2018

15. World Health Organization: WHA68.15: Strengthening Emergency and Essential Surgical Care and Anaesthesia as a Component of Universal Health Coverage. Geneva: WHO, 2015 ( http://apps.who.int/gb/ebwha/pdf_files/ WHA68/A68_R15-en.pdf) [Accessed November 25, 2019]

16. World Health Organization: WHA72.31: Emergency and Trauma Care: Emergency Care Systems for Universal Health Coverage: Ensuring Timely Care for the Acutely Ill and Injured. Geneva: WHO, 2019 ( https://apps.who.int/ gb/ebwha/pdf_files/WHA72/A72_31-en.pdf) [Accessed November 25,2019$]$

\section{Disclosures}

The authors report no conflict of interest concerning the materials or methods used in this study or the findings specified in this paper. The content is solely the responsibility of the authors and does not necessarily represent the official views of the National Institutes of Health.

\section{Author Contributions}

Conception and design: Garcia, Rosseau, Johnson, Lippa. Drafting the article: Park, Lippa. Critically revising the article: all authors. Reviewed submitted version of manuscript: all authors. Approved the final version of the manuscript on behalf of all authors: Garcia.

\section{Correspondence}

Roxanna M. Garcia: Northwestern University, Chicago, IL. roxanna.garcia@northwestern.edu; roxymgarcia@gmail.com. 